\title{
XIV.
}

\section{Weiterer Beitrag zur Klinik und Anatomie der Neuritis leprosa.}

(Aus den Hospital des Vaterländischen Frauen-Hülfs-Vereins.)

Von Dr. Ed. Arning und Dr. M. Nonne

in Hamburg.

(Hierzu Taf. VI-- VII.)

Am 14. Juni 1892 berichtete Einer von uns im Aerztlichen Verein zu Hamburg über einen Fall von generalisirter Lepra tuberosa $^{1}$ ); es waren während einer durch 18 Monate hindurch fortgesetzten klinischen Beobachtung an den Extremitäten keine jener Symptome zu constatiren gewesen, die man gewöhnlich bei peripherischer Neuritis findet: es hatten nehmlich Paresen, circumscripte Muskelatrophien, neuritische Sensibilitätsstörungen, Anomalien der elektrischen Erregbarkeit u. s. w. gefehlt; trotzdem ergab die mikroskopische Untersuchung, dass im N. ulnaris und im N. medianus an den "Prädilectionsstellen", oberhalb des Olecranon und am Handgelenk, hochgradige specifisch-lepröse neuritische und perineuritische Veränderungen Platz gegriffen hatten. Neben dieser Incongruenz der klinischen Symptome einerseits, den anatomischen Deformationen andererseits wurde noch eine auffallende Thatsache festgestellt, nehmlich das starke Zurücktreten der Waller'schen absteigenden Degeneration in den peripherischen Verzweigungen der an einzelnen Stellen ihres Verlaufs inteusiv degenerirten Nervenstämme. Der Eine von uns constatirte in der der Demonstration folgenden Discussion ${ }^{2}$ ), dass diese Thatsache in Einklang stehe mit seiner Auffassung, dass bei der Nervenlepra, nicht wie von Anderen angenommen werde,

1) Der Fall ist ausführlich publicirt: Jahrbücher der Hamburgischen StaatsKranken-Anstalten für 1892.

2) Neurol. Centralbl. 1892. S. 456 u. Deutsche Med. Wochenschr. 1893. No. XXII. S. 533 . 

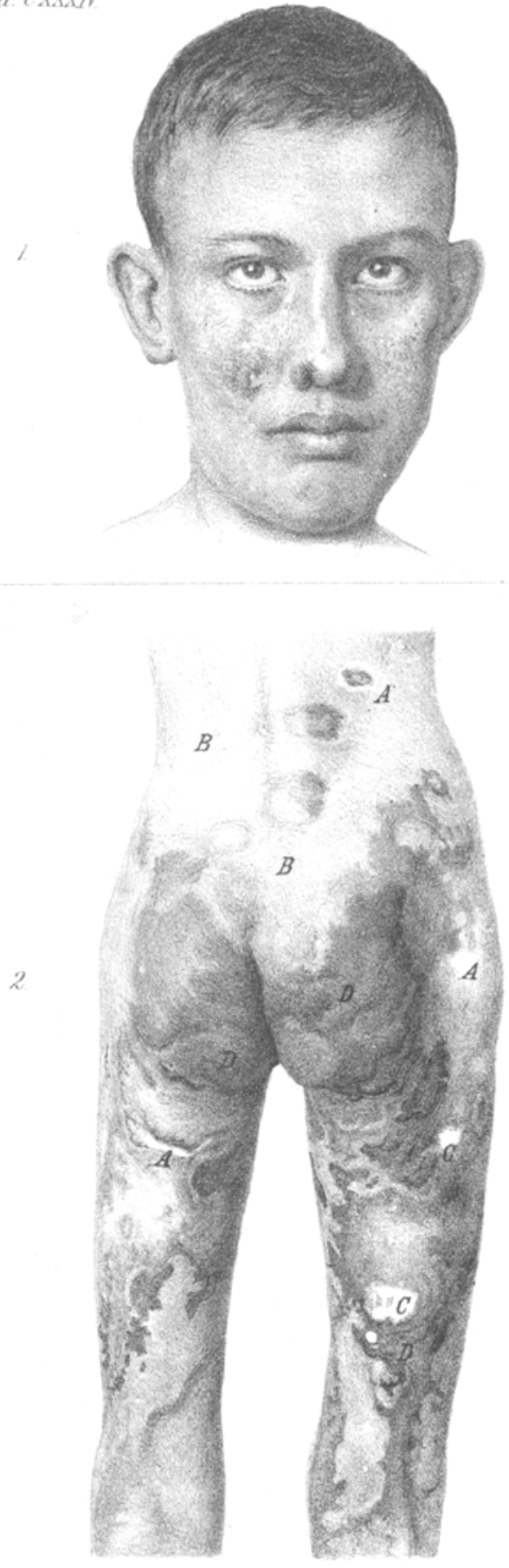

Mrb Stinitze Sith Snot. Borliw. 
3.

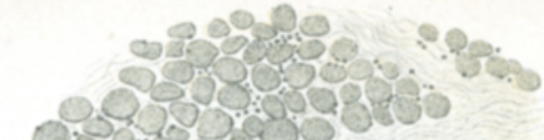

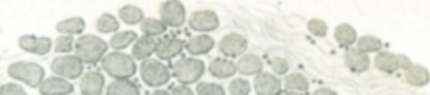 P.}

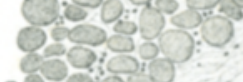

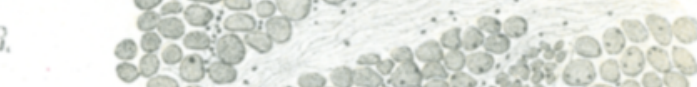
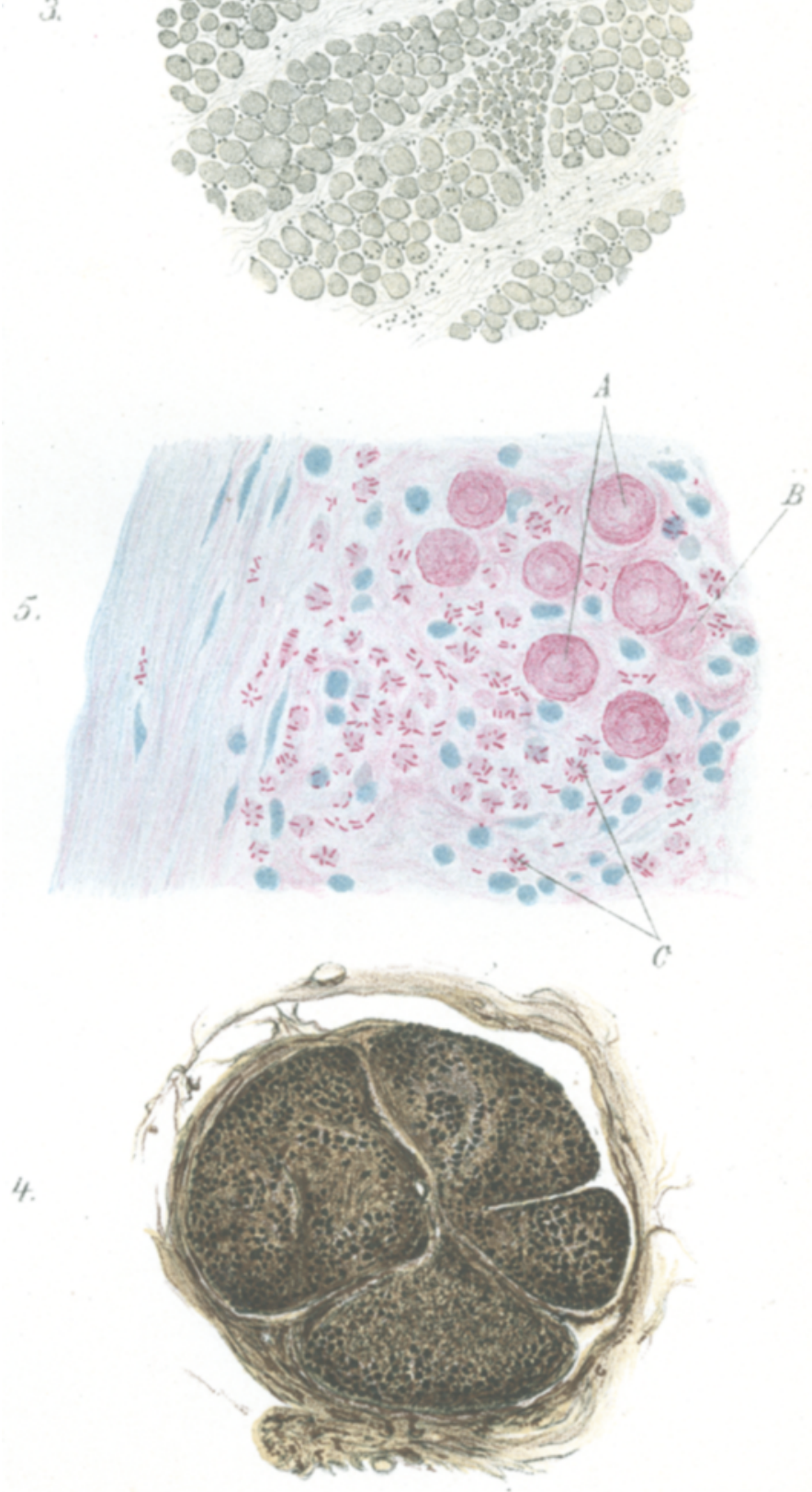

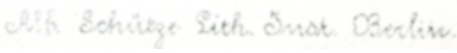


die Störungen der Senvibilität, Pigmentation u. s. w. auf sinen primären bacillaren Prozess in den Nervenendigungen der Haut zu beziehen seien, dass auch die Muskeln nicht primär bacillär erkranken, sondern dass sie langsam, parallel mit der Atrophie der. Haut, der Knorpel und des Knochengerüstes, schwinden, dass aber diese Muskeln bis zu ihrer vollständigen Atrophie eine zwar in der Gesammtwirkung schwächere, aber immerhin prompte und dem Witlensimpuls zuverlässig gehorchende Action bewahren.

Unna betonte bei dieser Gelegenheit, dass von klinischer seite bereits ofter die Erfahrung gemacht sei, , dass bei Fleckenlepra deutlichen Anschwellungen der Nerven oberhalb des Ellenbogengelenks keine entsprechenden Ausfälle an Nervenfunetion au der Peripherie (Hand and Pinger) entsprechen"; Unna sah das Neue in der von Nonne vorgetragenen Beobachtung darin, Iass sogar trotz Ravefaetion der Nervenstänme das Intactsein der. Nervenfunction bestehen könne; bisher habe man eben var ein Erhaltenbleiben von Nervenfasern trotz der leprösen Infltration angenommen.

Da die vorgetragenen Resultate immerhin höchst auffällig waren, so war es unser gemeinsames Bestreben, an einem geeigneten einschlägigen Falle von Neuem auf diese zwei Punkte 30 fahnden und dadurch dem Vorwurfe der Zufälligkeit, der gegen den obigen Fall von irgend einer Seite orhoben werden 落önte, zu begegnen.

Diese Gelegenheit bot sich uns im vergangenen Winter. In cie Behandlung des Einen von uns trat ein junger Mann, der wegen einer Erkrankung an Lepra Hülfe suchte.

Die Anamose ergab, dass derselbo im Jahre 1876 in Padagi auf Sunatra von curopäischen Eltern (Holländern) geboren war. Der Yater starb vor einigen Jahren an einem Herzleiden, die Mutter lebt und ist wach Angabe des Patienten gesund. Pat. ist der Jüngste von 12 Geschwistern, von denen 7 noch am Leben und gesund sind. Zwei altere Sehwestern des Pat., wine in llamburg, die andere in Amsterdam ansässig, zeigen nach eingehencer Untersuchnog keine Zeichen von Lepra. Nach Angabe dieser Schwestern war Pat. stets ein zartes Kind, bedeutend schwächer als seine Geschwister. in 13. Lebensjahre siedelte die Familie nach Penang in den Straits-Settlenents äber, wo viel Jepra herischt. Pat. spielte und badete dort mit eingebornen Kindern; von einew dieser Spielkameraden weiss man, dass er tepros war. In Jahre 1890 litt Pat. 4-5 Monate lang an einem an den Oberschenkeln localisirten Hautaussehlag. Es traten unter intensivem Jucken 
wasserbelle Blasen auf, welche zerkratzt $z u$ chronischen Geschwüren wurden, die schliesslich mit dünnen, blendend weissen, noch jetzt sichtbaren Narben abbeilten. Es hat sich da zweifellos um einen Pemphigus leprosus gehandelt, dem Ausdruck einer bereits fest etablirten Nervenlepra. Wir können somit sicher die Infection auf mindestens einige Jabre zurückdatiren. Nacbdem dieser Zustand etwa $\frac{1}{y} \mathbf{J}$ Jahr gewährt hatte, kam es zu häufigen Fieberanfällen, und während derselben bildeten sich, wesentlich an den Fxtremitäten, die typischen Ringe und Flecke der Lepra maculosa aus, ganz unabhängig von den oben erwähnten Narben. Die gyrirten Blecke waren zuerst schmal; mit ihrer allmählichen Verbreiterung ging Hand in Hand eine dunkelbraune Pigmentation und eine bedeutende Diekenzunahme der Haut in ihrem Bereiche. - Sehr bald stellten sich dann derbe knotige Einlagerungen in die Haut der Wangen und Nasenflügel ein, so dass Patient, der bis dahin stets auf Syphilis behandelt war, nach Europa geschickt wurde.

Er bot gleich beim ersten Anblick das klassische Bild der Lepra tuberomaculosa. Aus dem Status sei hervorgehoben, dass der langanfgeschossene Jüngling $47,5 \mathrm{~kg}$ wog, sich wohl und kräftig fühlte, nocb Keinen ausgesprochenen Leprageruch au sich hatte, in Bezng auf die Functionen seines Körpers mit Ausnahme der Haut sich normal verbielt, auch an den sichtbaren Schleimbäuten der Nase, der Mundböhle, des Rachens und des Kehlkopfes keine leprösen Veränderungen zeigte.

Die Untersuchung ergab ferner, dass die grösseren Hingeweide weder palpable Veränderungen noch Functionsstörungen zeigten. Der Urin war frei von Sediment, Eiweiss und Zueker. Hoden und Nebenhoden normal. Keinerlei Läbmungserscheinungen im Gesicht, auch die Augen und ihre Bedeckungen normal. Sehen, Bören, Schwecken und Riechen intact, in der sexuellen Spbäre keine Abnormitäten.

In Bezug auf die Vertheilung der leprösen Infiltrate der Haut sei im Wesentlichen auf die beigegebene Photographie des Gesichtes Fig. 1 und der Rückffäche des Körpers Fig. 2 verwiesen. - Die Vorderansicht bietet in Ganzen und Grossen ein gleiches Bild, und wie die Beine sind aueh die oberen Extremitäten befallen. Die Schwellungen der Wangen, der Nasenflügel sowie die beginnenden an den Ohren, sind eigentliche Leprome, $d . h$. mächtige Lager von Leprabacillen in der Cutis, ohne wesentliche Betheiligung des Nervensystems und ohne Pigmentation. Dementsprechend ist die Sensibilität an diesen Knoten nur sehr w'enig herabgesetzt. - Eben solche Lepraknoten befinden sich auf dem Dorsum der Kinger, hingegen bestehen solche eigentliche Leprome am Rumpf, und an Armen nnd Beinen nicht. Hier haben wir dunkel-leberbraune, zum Theil $3-5 \mathrm{~mm}$ hoeh aufliegende Flecken, eigentliche Lepride, $d$. h. Krankheitsprodulste, von welchen wir wissen, dass sie kleinzellige Infiltrate mit spärlichen Leprabacillen, nicht die mächtigen Anhäufungen grosser vacuolisirter bacillenbaltiger Zellen der Leprome darstellen. Bei diesen Lepriden ist anch die bei den Lepromen freie subepitheliale Schicht und das Epithel mit in den Krankheitsprozess bineingegezogen, was sicb durch die bis an's Rete heranreichende kleinzellige Infi- 
tration, die reichlicse Anwesenheit von Mastzes̆len und die Anhäufung ron Pigment in den Retezellen ausspricht. Dieser Anhüufung von Pigment entspricht eine Pigmentwerarmung im Centrum und, wie auf der Photographie bei Fig. 2 A ersichtlich, in der Peripherie der Flecken. Dass nicht nur das Centrum, sondern auch die Umgebung der Flecken und Ringe heller ist, als die noch normale Fant $B$, ist sonst im Allgemeinen ein ungewöbnlicber Befund bei Lepra, während diese Pigmentotaxis bei den Pigmentanomalien der Syphilis and der Vitiligo einen gewöbnlichen Befund darzustellen pflegt. Noch heller als diese pigmentarmen Stellet präsentiren sich die oben exwänten Narben des Pemphigus leprosus. Z Wei solche Stellen sind an der Hinterseite des linken Oberschenkels bei 0 deutlich sichtbar. Schliesslich sei als eigentbümlich die Mitbetheiligung der Haut des. Scrotum und des Penis erwähnt, welche im Allgemeinen nicht der Sitz lepröser Exantheme zu sein pflegen. - An dep afficirten Partien der Hant fehien die Lanugonärchen ${ }_{7}$ ebenso ist an denselben jede Schweisssecretion erloschen. Die llaare des Kopfes, die Angeabrauen und Wimpern, sowie die Achsel- und Sebamhaare sind gut erhalten.

Was die Sensibilität angeht, so ist dieselbe im Bereiche des Gesichtes nur unwesentlich herabgesetzt, da wie gesagt, auch auf den Lepromen alle Gefühlsqualitäten noch correct empfunden werden. Desgleichen zeigt die Conjunctiva palpebrarum et bulbi und die Cornea keine Anästhesie. Am Rumpfe findet sich eine ganz bedeutende Herabsetzung der Sensibilität an den Flecken und auch an solchen Stellen, wo keine deutliche Fleckenbildung za bemerken ist, wo jedoch das Aufhören der Sohweisssecretion und der Mangel des Auftretens einer Cutis anserina beil Reiz eine Ergriffonheit dieser Hautabschnitte erkennen lässt. Wesentlich stäker ausgesprochen ist der Verlust des Gefühls an den pigmentarmen Centren der Ringe und Guirlanten, sowie an den Weissen Varben (c) der Oberschenlsel. Hier existirt eine absolute Analgesie und ein vollständiger Mangel des Temperatursinns sowie der faradocutanen Sensibilität. - Tiefe, biş auf den Knochen geführte Nadelstiche werden nur als Druck und starke faradische Strome zwischen zwei aufgeselzten Nadelspitzen nur als Erschütterung gespürt. Dagegen lässt sich auch in diesem Falle die bei Lepra fast regehmäsig bemerkbare Thatsache constatiren, dass auch die leichteste Berührung, etwa mit einem lockeren Wattebausch oder mit einem Haarpinsel, correct und prompt erapfunden und localisirt wird. Deshalb ist es eigentlich auch nicht stricte correct, von einer Lepra anaesthetiea zu reden. Will man diese klinisehe Distinction an Stelle des besseren Ausdrucks Lepra nervorum beibehalten, so sollte man wenigstens den Terminus Lepra analgesica einfübren. An den Unterarmen und Händen, ebenso an den Unterschenkeln und Füssen ist die Analgesie sine allgemeine.

Patient warde behufs einer genatueren. Untersuchung des Nervensystems an 14. Januar 1893 in's Hospital des Vaterländischen Frauen-Hälfs-Vereins aufgenommen.

Bei derselben fanden wir:

Durchas keine spinaten oder cerebralen Symptome. Oberhalb des Ole- 
cranon und ain Handgelenk, an den von Alés her bekannten Prädilectionsstellen beiderseits, fühlt man eine mässig starke und mässig derbe spindelförmige Auftreibung des Nerwus ulnaris. Sämmtliche Bewegungen der Finger sind mit normaler Sebnelligkeit und in normaler Excursion ausfübrbar, auch die Extension der End-Phalangen des 2,-5. Fingers geschieht prompt. Die grobe Muskelkraft erscheint in den Mm. interossei (Spreizen und Schliessen der Finger, Hyperextension der Fingerspitzen) sowie in M. abduct. digiti $V$ beiderseits etwas herabgesetzt. Das Spat. inteross. I, weniger die Spat. inteross. II bis IV, erscheinen ganz leicht vertieft, was im Vergleich zu dem sonstigen Ernährungszustand des Kranken als pathologisch aufzufassen ist; ganz leicht abgeflacht ersebeint aucb der Hypothenar und vielleicht auch der Thenar; keine fibrillären Muskelzuckungen, keine sonstigen trophischen Störungen $\left.{ }^{1}\right)$ an der Haut und der Behaarung der Hand oder an den Fingernägeln. Betreffs des Verbaltens der Sensibilität sind oben bereits Angaben gemacht worden. Die Triceps- und Vorderarmreflexe sind schwach, aber deutlich erhalten.

Die u n teren Extremitäten verhalten sich nach jeder Richtung bin normal.

Die elektrische Erregbarkeit der oberen Extremitäten wurde mit Sorgfalt studirt, und zwar wurde sowohl die faradische wie die galvanische Erregbarkeit der Nerven und Muskeln zweimal untersucht.

Faradisch:

\begin{tabular}{|c|c|c|c|c|c|}
\hline Minimal-Contraction: & & Q.-A & stand & & \\
\hline & reel & & $\operatorname{lin!}$ & & \\
\hline N. accessorius & 110 & & 110 & $\mathrm{~mm}$ & \\
\hline N. axillaris & 80 & & 80 & - & \\
\hline Punctum Erb & 90 & - & 90 & - & \\
\hline N. ulnaris (Ellenb.) & . 100 & - & 95 & - & \\
\hline N. ulnaris (Handgel.) & 92 & - & 90 & - & \\
\hline N. medianus (Ellenb.) & 90 & - & 85 & - & \\
\hline M. cucullaris . . . & . 100 & - & 100 & - & \\
\hline M. deltoid. . . . & . 95 & - & 92 & - & \\
\hline M. biceps . . . . & 95 & . & 95 & - & \\
\hline II. triceps . . . . & $8 \check{a}$ & - & 85 & - & \\
\hline M. supinat. long. & 90 & - & 90 & - & \\
\hline M. extens. digit. comm. & 85 & - & 85 & - & \\
\hline M. abduct. poll. long. & 80 & - & 85 & - & \\
\hline M. flex. carpi ulnar. & 85 & - & 85 & - & \\
\hline M. flex. digit. commun. & 90 & - & 90 & - & \\
\hline Hypothenar. . . & 70 & - & 70 & - & \\
\hline Thenar . . . & 75 & - & 75 & - & \\
\hline M. inteross. I . & 82 & - & 82 & 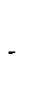 & $\left\{\begin{array}{l}\text { Contractionen irregulär, mitd. } \\
\text { W achsen des Stromes nicht } \\
\text { proport. an Stärke zunehmend. }\end{array}\right.$ \\
\hline M. inteross. II & $8 \overline{5}$ & - & 85 & - & \\
\hline M. inteross. III . . & 82 & - & 82 & . & \\
\hline
\end{tabular}

1) betreffs Lanugohärchen und Schweisssecretion vergl. oben. 
Gatranisub:

1. KaSz: N. accessorius

rechts

links

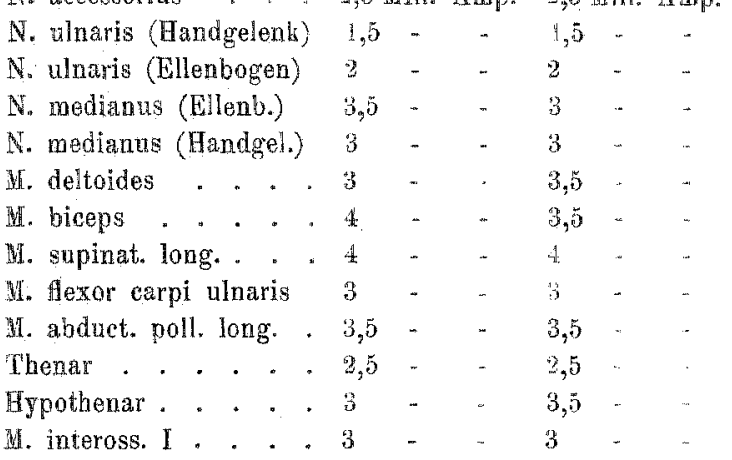

Die galvanischen Contractionen, sowohl die vom Nerv wie die von Muskel aus ausgelosten, sind durchweg kurz, überall überwiegt die KaSt die AnSZ; im M. inteross. I beiderseits besteht nur eine gewisse abnotme Wrmüdbarkeit, dergestalt, dass nach mehreren rasch hinter einander folgenden Reizen eine oder mehrere Contractionen ausfallen, ehe wieder eine Ántwort in Gestalt einer prompten Muskelzuckung auf die Reizung erfolgt.

Zusammengefasst sehen wir demnach, dass bei einem Leprösen, der deatliche spindelförmige Anschwellungen des Nervas ulnaris an den Prädilectionsstellen zeigt, die Nervenfunction der Hände und Finger nur in ganz geringem Grade gelitten hat: eine eben angedeutete Atrophie der Mm. interossei und eine ganz leichte Abflachung des Hypothenar und Thenar (?); die quantitative elektrische Erregbarkeit erscheint im Wesentlichen normal, für die Nerven sowohl wie für die Muskeln, und bei der qualitativen Untersuchung ergiebt sich nur für den M. inteross. I eine Anomalie in Gestalt einer gewissen Herabsetzung der Anspruchsfähigkeit auf rasch hinter einander folgende Reize; keine Anomalie der Zuckungsform und keine Umkehrung der Zuckungsformel.

Darüber, dass in den Nervenstämmen Lepröser dort, wo sie dom palpirenden Finger die charakteristische spindelförmige Anschwellung darbieten, hochgradige specifische Infiltrationen bestehen und ausgedehnte neuritische und perineuritische Veränderungen bereits zur Ausbildung gekommen sind, besteht durchaus kein Zweifel mehr, und hatte dies von Neuem der oben von uns citirte und im ärztlichen Verein vorgetragene Fall bewiesen; wir 
können somit auf Grund dieser ausnahmslosen Erfahrung auch für unseren Fall annehmen, dass eine starke Veränderung des Stammes des Nerv. ulnaris in der Mitte der Spindeln vorliegt.

Wir können demnach sagen, dass auch hier eine aufallende Incongruenz zu Tage tritt zwischen der Nervenfunction der vom Nerv. ulnaris abhängigen Partien der Hand und Finger einerseits, der leprösen Invasion des Nervenstammes andererseits.

Um zu einer Beantwortung der zweiten Frage zu kommen, wie es mit dem anatomischen Verhalten der Muskeln gegenüber dem Nervenstamme stehe, nahmen wir, nach vorher eingeholter Einwilligung des Kranken, eine Untersuchung von, dem Kranken in vivo excidirten Muskel- und Nervenstückchen vor. Herr Dr. Waitz, Oberarzt der chirurgischen Abtheilung des Hospitals des vaterländischen Frauen-Hülfsvereins, legte in Narkose und unter Esmarch'scher Blutleere sowohl am Olecranon wie am Handgelenke linkerseits den Stamm des Nerv. ulnaris in einer Ausdehnung von $4 \mathrm{~cm}$ frei. Es wurde eine sehr deutliche spindelförmige Auftreibung des etwas graulichen und wie getrübt aussehenden Nervenstammes constatirt; vom untersten Ende der "Spindel" wurde sowohl am Olccranon als am Handgelenk ein kleines Stück vom medialen Rande des Nervenstammes excidirt; an der Stelle, an der die Excision vorgenommen wurde, sah der Nerv weder grau noch "getrübt" aus. Ebenso excidirten wir aus der Mitte des von Herrn Dr. Waitz freigelegten Hypothenar und M. interosseus I sin. je ein Stückchen; der freigelegte Muskel sah makroskopisch normal aus.

Ein Partikel der zwei Muskelstückchen wurde frisch in $\frac{3}{4}$ procentiger Kochsalzlösung, ein anderes Partikelchen untersucht, nachdem es 24 Stunden in Sol. Müller gelegen hatte. Der Rest wurde in Sol. Müller gehärtet (3 Monate), in Alkohol nachgehärtet, in Celloidin eingebettet und an gefärbten Schnittpräparaten untersucht.

Je ein Partikel der excidirten Nervenstücke wurde in 1procentiger Osmiumsäure 24 Stunden gefärbt, zerzupft und in Liq. Kali acet. untersucht; der Rest der 2 Nervenstücke wurde wie die 2 Muskelstücke gehärtet und eingebettet.

Die frische Untersuchung (Zerzapfung) des Muskels und die- 
jenige des kuze Zeit in Nuller'seher Flüssigkeit gewesenen Muskels ergab, um es kurz abzumachen, weder am Parenchym, noch an den Kernen, noch am interstitiellen Gewebe irgend eine bemerkenswerthe Anomalie; obenso sahen wir an den zerzupfton und mit 1procentiger Osmiumsäure gefürbten Nervenfasern aussohliesslich normale Verhältnisse. Herr Dr. Eisenlohr, welcher die Freundlichkeit hatte, diese Präparate durchzusehen, konnte auch keine pathologischen Verhältnisse finden.

Die Schnitte der gehärteten Muskelstücke wurden zunächst mit Heidelberger Alaun-Carmin und dann nach der von Unna angegebenen Methode, die dem Einen yon uns bei seinen letzten Untersuchungen auf Leprabacillen die besten Resultate ergeben hatte - Färbung mit Carbolfuchsin, Entfärbung in 20 procentigen Salpetersäure-Alkobol, Nachfärbung mit concenteirter wässeriger Methylenblanlösung - gefärbt.

Es fanden sich im M. hypothenar (abducens digiti V) in den untersuchten Quer- und Längsschnitten ausschliesslich normale Verhälnisse, d. h. an den Alaun-Carminpräparaten sah man weder vereinzelte noch gruppenweise angehäufte verschmälerte Vuskelfasern, keine Wucherung des interstitiellen Bindegewebes, keine "Kernzeilen" an Längsschnitten, an den normal runden bezw. 4-5-6eckigen Querschnittsbildern der Muskelfasern durchschnittlich 4-8 normale Kerne, keine hypertrophischen Muskelfasern; die hie und da sich präsentirenden intramusculären Nervenfasern zeigten pormale Verhältnisse; neuromusculäre Stämmchen - eine, wie jetat bekannt, nicht pathologische Erscheinung ${ }^{1}$ ) - kamen mässig zahlreich zu Gesicht.

Auch an den kleinen Gefüssen waren die Wandverhältnisse normal. An den nach Unna's Methode behandelten Präparaten fanden sich keine Leprabacillen, weder im Muskelparenohym, noch im Perimysium, weder in den Waudungen der

Diese Gebilde, die von Eichhorst s. Z. (dieses Archiv Bd. 112, H. 2) als Produkte einer "Neuritis fascians" beschrieben wurden, und die vor Eichhorst anch von Eisonlobr und von E. Fränkel geschildert und als "umschnürte Bündel" bezeichnet waren, sind durch die Nachuntersuchungen von Siemerling (Westphal's Archiv 1888) und Nonne (Zeitschr. f. klin. Med. Bd. 15 , H. 5 u. 6) als reguläre Vorsommnisse festgestellt. 
quer getroffenen kleinen Gefässe, noch an den intramusculären Nervenzweigchen.

Im Musc. inteross. I (s. Fig. 3) bestanden im Grossen und Ganzen dieselben normalen Verhältnisse, nur tauchten, wenn auch recht vereinzelt und weit aus einander liegend, hie und da ganz kleine Inseln einfach-atrophischer Fasern auf; das Volumen dieser atrophischen Fasern zeigte sich auf etwa $\frac{1}{4}-\frac{1}{5}$ der normalen Breite reducirt; dabei war die Querstreifung, wie die Durchsicht von Längsschnitten ergab, normal erhalten, und die Kerne erschienen nur relativ vermehrt, weil einander näher gerückt; von einer nennenswerthen Zunahme des interstitiellen Bindegewebes war keine Rede, auch hier konnten wir an den - allerdings sehr spärlich zu Gesicht kommenden - kleinen intramusculären Nervenzweigen keine Degenerationen sehen, und auch hier erschienen die Gefässwandungen nicht verdickt. Die Untersuchung dieser Muskelpräparate auf Leprabacillen fiel ebenso negativ aus wie die der Präparatedes Musc. abductor digiti $V$.

Durchaus positiv war dem gegenüber das Ergebniss der Untersuchung der gebärteten Nervenstïcke. Dieselben wurden zunächst mit Borax-Carmin, sowie nach Weigert's Hämatoxylinmethode gefärbt und in zweiter Linje nach Unna's Methode untersucht.

Das Stück, welches rom untersten Ende der Spindel am Olecranon excidirt war, zeigte (s. Fig. 4) ziemlich hochgradige Degenerationen; in einer Reihe von Nervenbündelchen waren alle Fasern intact, in anderen waren nur wenige Fasern degenerirt, in der Mehrzahl der Bündel jedoch überwog die Anzahl der degenerirten Fasern die der normalen; in diesen stärker degenerirten Bündeln war entweder ein amorphes Gewebe an Stelle der Nervenfasern getreten, oder ein bienenwaben- bezw. netzartiges Maschenwerk, in dem nur noch hie und da eine theilweise oder bereits ganz zerstörte Nervenfaser hing, hatte die Querschnitte der Nervenröhren ersetzt. Die zwiebelschalenförmigen concentrischen Gebilde, die bei dem Falle, der im ärztlichen Verein demonstrirt wurde, so prägnant zu Tage traten und die den Nervenquerschnitt fast wie von einer Neubildung befallen erscheinen liessen, - Neubildungen, die als endoneurale aufgefasst wurden 
uad den in nenester Zeit viel beschriebonen Raynaat'sohen Körperchen gleichgestellt wurden - fehlten hier vollig; ebenso lagen keine eigentlichen perineuritischen Veranderungen vor. Die Gefässe waren wohl nicht gane normal, wenigstens erschion die Media an den grösseren Arterien etwas breit, ohne aber weitere Veränderungen, wenigstens für die zwei genannten Farbenreactionen, aufzuweisen. Alles in Allem liess sich also eine mässig hochgradige Faserrarefication in einer Reihe von Bündeln, in der Minderzahl der Bïndel eine starke Faserdegeneration naohweisen.

Die auf Bacillen gefärbten Präparate zeigten, dass iiber den ganzen Querschnitt verstrent, und speciell auch iberall zwischen den normalen Markfasern vereinzelte Leprabacillen lagen; die so typischen Bacillenhaufen, mag man sie nun als Leprabacillen uder lediglich als Klumpen massenhafter Bacillen aufassen, sahen wir nirgends; in der Wandung der Gefüsse fanden wir keine Bacillen. Sehr bemerkenswerth war ferner noch ein. Befund: anitten in einzelnen quorgeschnittenen Nervenfasern, die daulurch, dass sie die blaue Ueberfarbung angenommen hatten, sich als degenerirt documentirten, sah man charakteristische Leprabacillen; es war uns unzweifelhaft, dass an diesen Stellen die specifischen Bacillen wirklich die Nervenfasem selbst befallen hatten, eine Thatsache, die bekanntlich bisher von verschiedenen Seiten noch angezweifelt wurde ${ }^{1}$ ) (s. Fig, 4). Ganz dieselben Verhältnisse, was den Charakter der Degoneration anbetrifft, zeigten sich in tem der Nervenspindel am Handgelenk excidirten Stück, nur waren hier die Bündel, in denen eine starke Degeneration über đen grösseren Theil des Querschnitts sich nachweisen liess, spärlicher. Der Bacillenbefund war der gleiche wie am Ellenbogen.

Es hat sich sonach ergeben, dass am unteren Ende der wie wir anzunehmen berechtigt sind - in ihrer Mitte stark degenerirten Spindel des Nerv. ulnaris erhebliche Alterationen Statt haben, die jedoch nicht dem Grade entsprechen, wie wir

YGanz netuerdings haben (Nouv. iconogt, de la Sulpetrière, 1893, No. 3) Pitres und Sabrazès denselben Bacillenbefund an einem intra vitan exeidirten Lepraners erhobea; das blinische Bild des Falles von P. nnd S. war das einer Syringomyelie; erst der mikroskopische Befund liess die richtige Diagnose stellen. 
sie dicht unterhalb der durch andere Noxen neuritisch degenerirten Nervenpartien zu sehen gewohnt sind; in den intramuseuIären Nerven fehlten nachweisliche Degenerationen, die Muskeln waren - M. hypothenar - entweder normal, oder - M. interosseus I - nur an vereinzelten Stellen einfach atrophisch. Somit haben wir auch in anatomischer Hinsicht hier im Wesentlichen das gleiche. Resultat wie in dem früher von Nonne beschriebenen und hier schon öfter citirten Falle, d. h. wir sehen auch hier, dass bei leprösen Nerven ein Missverhältniss bestehen kann zwischen peripheriseh gelegenen Nerv-Muskelfasern und mehr proximal localisirter Degeneration.

Die frische Untersuchung allein hätte uns za der irrthümlichen Annahme verleiten können, dass jede Degeneration fehle: ein Blick auf die Querschnittsbilder der Nerven und Muskeln belehrt uns, dass bei beliebig hinausgezupften Partien in der That pathologische Fasern fehlen können, und dass nur ein Ueberblick äber ein zusammenhängendes Querschnitts- oder Längsschnittsfeld diese pathologischen Fasern zu Gesicht bringt.

Das Ergebniss unserer Untersuchungen ist, zusammengefasst, somit folgendes: Bei der Neuritis und Perineuritis leprosa, welche die Nervenstämme an den Prädilectionsstellen hochgradig zerstört, leisten die unterhalb dieser typischen Stellen gelegenen Verzweigungen der Nerven viel länger Widerstand als bei anderen zu anatomischen Degenerationen einzelner Stellen der Nervenstränge führenden Schädlichkeiten; die Leprabacillen finden sich schon disseminirt da, wo die Degeneration der Nervenfasern noch fehlt oder erst ganz sporadisch sich zeigt; wo die secundäre - absteigende - Degeneration auftritt, befällt sie nicht den Gesammtquerschnitt, sondern schont eine mehr oder weniger grosse Anzahl von Bündelchen; dementsprechend ist das anatomische Verhalten der Muskeln selbst bei hochgradiger lepröser Veränderung ihrer zugehörigen Nervenstämme an den "Orten der Wahl" lange Zeit - entsprechend dem Verhalten der an die Muskeln tretenden Nervenfasern - entweder ein ganz normales, oder die Atrophie der Muskelfasern ist auf nur einzelne Bündelchen beschränkt; es geht somit das klinische Bild der Function der peripherischen Theile der Extremitäten mit den anatomisch an den ausserhalb 
des Bereichs der Spindel-Auftreibung der Nerven za constatirenden Thatsachen Hand in Hand.

Es ist selbstverständlich, dass diese Incongruenz $z$ wischen den hochgradig leprös veränderten Nervenstrecken und den peripherischen Nerven-Partien einerseits, die Incongruenz zwischen dem klinischen Bilde und diesen eben genannten anatomischen Degenerationen andererseits auch nur die Frage der - wenn auch auffallend langdauernden - Zeit ist; schliesslich verfallt die peripherische Nervenstrecke und der ihr zugehörige Muskelbezirk doch dem Untergang und zwar durch den deletären Einfluss der hier direct befallenden Leprabacillen einerseits, auf dem Wege der secundären Degeneration andererseits, und wir sehen dann schliesslich auch den klinischen Ausdruck derselben, der, von Neuritiden anderen Herkommens aus geläufig, auch für die lepra neuerdings, zuletzt von Schultze (Deutsches Archiv 1. klin. Med. Bd. 43) geschildert worden ist.

\section{Erklårung der Abbildungen. \\ Tafel und VIr.}

Fig. Photographia des Gesichtes, Leprome an Wangen, Nase und Ohren zeigend.

Hig. 2. Rückenansicht des Patiented. A pigmentarme Havt im Centrum und in der Peripherie der Lepride. B normale Maut. C blendend weisse Narben vom Pemphigus leprosus. O) die grossen brannen thepride.

Fig. 3. Twischen den normalen Muskelquerschnitten zeigt sich, vereinzelt auftauchend, ein kleines Fold atrophischer Muskelfasern; keine als pathologisch anzusprechende Kernwucherung.

Fig. \& zeigt die Ausdehnung der Degenerationen am N. unar. arn WeigertPräparat; die Intensität des Faserschwundes ist eine sehr verscbiedene. (Zeiss, Ocular 1, Linse AA.)

Tig. 5 zeigt eine Partie von derselben Nervenpartie wie Fig. 4 bei stärberer Yergrösserung (Zeiss, Ocular 2, Oelimmers. $\frac{1}{12}$ ) und Bacillenfärbung. A normale Nervenfasern, documentirt durch Grösse und Bestand der Tuchsinfärbung (Säurefestigkeit). B beginnende Bakterieninvasion in den Nerven, Grôssen - und Farbenschwund. O durch Bacillen zerstörte Nervenfaserv. Geringe Betheiligung des Perineurium. 